Bull. Austral. Math. Soc.

$46 \mathrm{~B} 20,46 \mathrm{E} 30$

VOL. 54 (1996) [431-440]

\title{
ON THE WEAKLY STRONGLY EXPOSED PROPERTY AND SOME SMOOTHNESS PROPERTIES OF ORLICZ SPACES
}

\author{
Yunan Cui, Henry K. Hudzik and Hongwei Zhu
}

The notion of a weakly strongly exposed Banach space is introduced and it is shown that this property is the dual property of very smoothness. Criteria for this property in Orlicz function spaces equipped with the Orlicz norm are presented. Criteria for strong smoothness and very smoothness of their subspaces of order continuous elements in the case of the Luxemburg norm are also given.

\section{O. INTRODUCTION}

Throughout this paper $X$ denotes a Banach space, $X^{*}$ denotes its dual space, $S(X)$ and $S\left(X^{*}\right)$ denote respectively the unit spheres of $X$ and $X^{*}$. The triple $(G, \Sigma, \mu)$ stands for a nonatomic, complete and finite measure space and $L^{0}$ stands for the space of all (equivalence classes of) $\Sigma$-measurable real functions defined on $G$.

By $\Phi$ we denote an Orlicz function, that is, $\Phi$ is defined on the real line $\mathbb{R}$ with values in $\mathbb{R}_{+}=[0,+\infty)$ and it vanishes only at zero, it is even, convex with $\Phi(u) / u \rightarrow 0$ as $u \rightarrow 0$ and $\Phi(u) / u \rightarrow+\infty$ as $u \rightarrow+\infty$.

For any Orlicz function $\Phi$ we define on $L^{0}$ the modular $I_{\Phi}$ by

$$
I_{\Phi}(x)=\int_{G} \Phi(x(t)) d \mu .
$$

The Orlicz space $L^{\Phi}$ (or $L_{0}^{\Phi}$ ) is the set

$$
\left\{x \in L^{0}: I_{\Phi}(\lambda x)<+\infty \quad \text { for some } \lambda>0\right\}
$$

equipped with the Luxemburg norm

$$
\|x\|=\inf \left\{\lambda>0: I_{\Phi}(x / \lambda) \leqq 1\right\}
$$

(or with the Orlicz norm)

$$
\|x\|^{0}=\sup \left\{\int_{G} x(t) y(t) d \mu: y \in L^{\Psi}, I_{\Psi}(y) \leqq 1\right\}
$$

Received 21st December 1995

Y. Cui was supported by a Chinese National Science Foundation Grant and H. Hudzik was supported by KBN Grant 2PO3A 03110.

Copyright Clearance Centre, Inc. Serial-fee code: 0004-9729/96 \$A2.00+0.00. 
where $\Psi$ denotes the function complementary to $\Phi$ in the sense of Young, that is,

$$
\Psi(u)=\sup \{u v-\Phi(v): v \geqq 0\}
$$

(see $[7,8,8,13])$.

$E^{\Phi}$ (or $E_{0}^{\Phi}$ ) denotes the subspace of $L^{\Phi}$ (or $L_{0}^{\Phi}$ ) defined by

$$
E^{\Phi}=\left\{x \in L^{0}: I_{\Phi}(\lambda x)<+\infty \text { for any } \lambda>0\right\}
$$

and equipped with the norm induced from $L^{\Phi}$ (or $L_{0}^{\Phi}$ ).

It is more convenient to use the Amemiya formula for the Orlicz norm:

$$
\|x\|^{0}=\inf \left\{\frac{1}{k}\left(1+I_{\Phi}(k x)\right): k>0\right\}
$$

which does not use the complementary function of $\Phi$ (see [7] and [13]). The set of all $k>0$ for which the infimum in the Amemiya formula for $x \in L^{\Phi} \backslash\{0\}$ is attained will be denoted by $K_{\Phi}(x)$. It is known that $K_{\Phi}(x) \neq \emptyset$ for any $x \in L^{\Phi} \backslash\{0\}$, since $\Phi(u) / u \rightarrow+\infty$ as $u \rightarrow+\infty$ (see [6]).

We say an Orlicz function $\Phi$ satisfies the $\Delta_{2}$-condition ( $\Phi \in \Delta_{2}$ for short) if there exist positive constants $K$ and $u_{0}$ such that $\Phi(2 u) \leqslant K \Phi(u)$ whenever $|u| \geqslant u_{0}$ (see [7] and [13]). It is obvious that the definition of the $\Delta_{2}$-condition does not change if we replace the number 2 by any $\lambda>1$.

Let us recall now some geometric notions. A Banach space $X$ is said to be weakly locally uniformly rotund ( $W L U R$ for short) if for each $x \in S(X)$ and each sequence $\left\{x_{n}\right\}$ in $S(X)$ such that $\left\|x_{n}+x\right\| \rightarrow 2$, we have $x_{n} \rightarrow x$ weakly ( $x_{n} \stackrel{w}{\rightarrow} x$ for short), see [5]. $X$ is said to have property $W M$ if for any $x \in S(X)$ and any sequence $\left\{x_{n}\right\}$ in $S(X)$ such that $\left\|x_{n}+x\right\| \rightarrow 2$ there exist a support functional $f_{x}$ at $x$ and a subsequence $\left\{x_{n_{i}}\right\}$ such that $f_{x}\left(x_{n_{i}}\right) \rightarrow 1$ (see $[3,10,11]$ ). Recall that $f_{x} \in X^{*}$ is called a support functional at $x \in X \backslash\{0\}$ if $\left\|f_{x}\right\|=1$ and $f_{x}(x)=\|x\|$ (see [12]). The set of all support functionals at $x$ is denoted by $\operatorname{Grad}(x)$. It is obvious that $W L U R$ Banach spaces have property $W M$. The question arises as to whether or not there is some property (denote it for the moment by $A$ ) such that $W M$ and $A$ are together equivalent to $W L U R$. The answer is affirmative. This leads us to the notion of weakly strongly exposed (WSE for short) Banach spaces. A Banach space $X$ is said to be weakly strongly exposed if for any $x \in S(X)$ and any $\left\{x_{n}\right\}$ in $S(X)$, if $f_{x}\left(x_{n}\right) \rightarrow 1$ for some $f_{x} \in \operatorname{Grad}(x)$ then $x_{n} \stackrel{w}{\rightarrow} x$. As we shall see, this notion is dual to very smoothness.

A Banach space $X$ is called smooth if for any $x \in S(X)$ there is exactly one element in Grad $(x) . X$ is said to be very smooth (strongly smooth) if it is smooth 
and for each $x \in S(X)$ and each $\left\{f_{n}\right\}$ in $S\left(X^{*}\right)$ such that $f_{n}(x) \rightarrow 1$, we have $f_{n} \stackrel{w}{\rightarrow} f_{x}\left(\left\|f_{n}-f_{x}\right\| \rightarrow 0\right)$, where $\left\{f_{x}\right\}=\operatorname{Grad}(x)$. We denote these properties for short by $V S$ and $S S$, respectively.

The rest of the paper will be divided into two parts. In the first part we shall give some general results on the properties $W M, W L U R, W S E$ and $V S$. In the second one we shall present criteria for $V S$ and $S S$ of $E^{\Phi}$ and for the property $W S E$ in $L_{0}^{\Phi}$. Let us recall that criteria for $V S$ and $S S$ of $L_{0}^{\Phi}$ were given in [2]. To get these criteria, some duality arguments were used. Since $E^{\Phi}$ need not be a dual space, it was necessary to find a new method to get an analogue of the results from [2] for $E^{\Phi}$ in place of $L_{0}^{\Phi}$.

\section{SOME GENERAL RESUlts}

Let us start with the following theorem.

Theorem 1.1. If $X^{*}$ is $V S$, then $X$ is $W S E$.

Proof: Let $x \in S(X)$ and $\left\{x_{n}\right\}$ in $S(X)$ be such that $f_{x}\left(x_{n}\right) \rightarrow 1$ for some $f_{z} \in \operatorname{Grad}(x)$. Since $V S$ of $X^{*}$ yields reflexivity of $X$ there are a subsequence $\left\{x_{n_{i}}\right\}$ of $\left\{x_{n}\right\}$ and $x^{\prime} \in S(X)$ such that $x_{n_{i}} \stackrel{w}{\rightarrow} x^{\prime}$. In particular, $f_{x}\left(x_{n_{i}}\right) \rightarrow f_{x}\left(x^{\prime}\right)$ and, by the uniqueness of the limit, we get $f_{x}\left(x^{\prime}\right)=1$. Therefore, $f_{x}\left(x+x^{\prime}\right)=2$ and consequently $\left\|x+x^{\prime}\right\|=2$. Since $V S$ of $X^{*}$ implies rotundity of $X$, we get $x^{\prime}=x$. Therefore, $x_{n_{i}} \rightarrow x$ and by the double extract subsequence theorem, $x_{n} \rightarrow x$. This means that $X$ is $W L U R$.

Theorem 1.2. If $X^{*}$ is $W S E$, then $X$ is $V S$.

Proof: Let $X^{*}$ be WSE, $x \in S(X),\left\{f_{n}\right\}$ be in $S\left(X^{*}\right)$ and $f_{n}(x) \rightarrow 1$. By the Hahn-Banach theorem there is $f_{x} \in \operatorname{Grad}(x)$, that is, $\left\|f_{x}\right\|=f_{x}(x)=\|x\|=1$. Taking into account that $X$ is canonically embedded into $X^{* *}$ we can write $x\left(f_{n}\right) \rightarrow 1=x\left(f_{z}\right)$ so that $x \in \operatorname{Grad}\left(f_{x}\right)$, again identifying $x$ with its embedding. Now, by the assumption that $X^{*}$ is $W S E$ we get $f_{x} \stackrel{w}{\rightarrow} f_{x}$, which completes the proof.

Theorem 1.3. $X$ is $W L U R$ if and only if $X$ has property $W M$ and $X$ is $W S E$

Proof: The necessity is obvious. To prove the sufficiency, assume that $X$ has both properties $W M$ and $W S E$ and that $x \in S(X),\left\{x_{n}\right\}$ is a sequence in $S(X)$ and $\left\|x_{n}+x\right\| \rightarrow 2$. Since $X$ has property $W M$ there exist a subsequence $\left\{x_{n_{i}}\right\}$ of $\left\{x_{n}\right\}$ and some $f_{z} \in \operatorname{Grad}(x)$ such that $f_{x}\left(x_{n_{i}}\right) \rightarrow 1$. Since $X$ is $W S E$, this implies that $x_{n_{i}} \stackrel{w}{\rightarrow} x$ and, by the double extract subsequence theorem, we get $x_{n} \stackrel{w}{\rightarrow} x$. 
2. VERY SMOOTHNESS AND STRONG SMOOTHNESS OF $E^{\Phi}$ AND PROPERTY $W S E$ IN $L_{0}^{\Phi}$.

THEOREM 2.1. For the space $E^{\Phi}$ the following statements are equivalent:

(1) $E^{\Phi}$ is strongly smooth,

(2) $E^{\Phi}$ is very smooth,

(3) $\Phi$ is smooth and $\Psi \in \Delta_{2}$.

ProOF: The implication (1) $\Rightarrow(2)$ is obvious. We shall prove now that $(2) \Rightarrow$ (3). Since very smoothness implies smoothness, we conclude that $\Phi$ must be smooth on $\mathbb{R}$ (see $[1,6,14])$. So, we only need to prove that very smoothness of $E^{\Phi}$ implies that $\Psi \in \Delta_{2}$. Otherwise, we have $E_{0}^{\Psi} \subsetneq L_{0}^{\Psi}$. By the Bishop-Phelps theorem there is $f \in S\left(L_{0}^{\Psi}\right) \backslash S\left(E_{0}^{\Psi}\right)$ and $x \in S\left(E^{\Phi}\right)$ such that $f(x)=\|f\|^{0}=1$, where $f(x)$ stands for $\langle x, f\rangle$. Define $f_{n}(t)=f(t) \chi G_{n}(t)$, where $G_{n}=\{t \in G:|f(t)| \leqq n\}$. Then

$$
f_{n}(x)=f(x)-\int_{G \backslash G_{n}} f(t) x(t) d \mu .
$$

Since the norm in $E^{\Phi}$ is absolutely continuous, we have

$$
\lim _{n \rightarrow \infty}\left|\int_{G \backslash G_{n}} f(t) x(t) d \mu\right| \leqq \lim _{n \rightarrow \infty}\|f\|^{0}\left\|x \chi_{G \backslash G_{n}}\right\|=0
$$

that is, $f_{n}(x) \rightarrow 1$. Since $f \in S\left(L_{0}^{\Psi}\right) \backslash S\left(E_{0}^{\Psi}\right)$, by the Hahn-Banach theorem there is a singular functional $s \neq 0$ such that $s(f) \neq 0$. Since $f_{n} \in E_{0}^{\Psi}$, we have $s\left(f_{n}\right)=0$. Hence $E^{\Phi}$ is not very smooth, a contradicition which completes the proof.

$(3) \Rightarrow(1)$. For each $x \in S\left(E^{\Phi}\right)$ and $\left\{f_{n}\right\}$ in $S\left(E_{0}^{\Psi}\right)$ such that $f_{n}(x) \rightarrow 1=f(x)$ we shall prove that $\left\|f_{n}-f\right\|_{\Psi}^{0} \rightarrow 0$, where $f$ is the unique support functional at $x$ (by smoothness of $E^{\Phi}$, which follows by smoothness of $\Phi$, see [6]). Let $k_{n} \geqq$ $1(n=0,1,2, \ldots)$ be such that

$$
\left\|f_{n}\right\|^{0}=\frac{1}{k_{n}}\left(1+I_{\Psi}\left(k_{n} f_{n}\right)\right), \quad\|f\|^{0}=\frac{1}{k_{0}}\left(1+I_{\Psi}\left(k_{0} f\right)\right) .
$$

The existence of such constants $k_{n}$ follows since $(\Phi(u) / u) \rightarrow+\infty$ as $u \rightarrow+\infty$ (see [6]). We shall present our proof in three steps.

I. We shall prove that $\left\{k_{n}\right\}_{n=0}^{\infty}$ is a bounded sequence.

(i) Since $\left(E^{\Phi}\right)^{*}=E_{0}^{\Psi}$, there is a subsequence $\left\{f_{n_{i}}\right\}$ of $\left\{f_{n}\right\}$ and $f^{\prime} \in E_{0}^{\Psi}$ such that $f_{n_{i}} \stackrel{E^{\Phi}}{\rightarrow} f^{\prime}$. By $f_{n}(x) \rightarrow 1$ and $x \in E^{\Phi}$, we have. $f_{n_{i}}(x) \rightarrow 1$, whence $f^{\prime}(x)=f(x)$. Since $E^{\Phi}$ is smooth, we get $f=f^{\prime}$ and so $f_{n} \stackrel{E^{\Phi}}{\rightarrow} f$. 
(ii) We shall prove that $\left|f_{n}\right| \stackrel{E^{\Phi}}{\rightarrow}|f|$. From

$$
1=f(x) \leqslant|f|(|x|) \leqq\|f\|^{0}\|x\|=1
$$

we get $|f|(|x|)=1$. By the same argument as in (i), we obtain $\left|f_{n}\right| \stackrel{E^{\Phi}}{\rightarrow}|f|$.

(iii) We shall show that there exist $a>0, \varepsilon>0$ and $n_{0} \in \mathbb{N}$ such that

$$
\mu\left\{t \in G:\left|f_{n}(t)\right| \geqq a\right\}>\varepsilon \quad \text { whenever } \quad n \geqq n_{0} .
$$

Define $\alpha=\int_{G}|f(t)=| d \mu$ and take $0<\beta<\alpha / 3 \mu(G)$. By $I_{\Psi}\left(f_{n}\right) \leqq 1$ and the ValléePoussin theorem, the functions of the sequence $\left\{\left|f_{n}\right|\right\}$ have equi-absolutely continuous integrals (see [7] and [13]). So, for $\varepsilon_{0}=\alpha / 3$ there is a $\delta>0$ such that

$$
\int_{F}\left|f_{n}(t)\right| d \mu<\varepsilon_{0} \quad \text { whenever } \quad \mu(F)<\delta .
$$

If (1) does not hold, then for each $\beta>0$ and $n \in \mathbb{N}$ there is $n_{i} \in \mathbb{N}$ such that $\mu\left\{t \in G:\left|f_{n_{i}}(t)\right| \geqq \beta\right\}<\delta$. Therefore,

$$
\begin{aligned}
\int_{G}\left|f_{n_{i}}(t)\right| d \mu & =\int_{G \backslash\left\{t \in G:\left|f_{n_{i}}(t)\right| \geqq \beta\right\}}\left|f_{n_{i}}(t)\right| d \mu+\int_{\left\{t \in G:\left|f_{n_{i}}(t)\right| \geqq \beta\right\}}\left|f_{n_{i}}(t)\right| d \mu \\
& <\beta \mu(G)+\varepsilon_{0}<\frac{2}{3} \alpha
\end{aligned}
$$

which contradicts (ii).

If the sequence $\left\{k_{n}\right\}$ is not bounded, there is a subsequence $\left\{k_{n_{i}}\right\}$ of $\left\{k_{n}\right\}$ such that $\lim _{i \rightarrow \infty} k_{n_{i}}=\infty$. Hence

$$
\begin{aligned}
1 & =\left\|f_{n_{i}}\right\|^{0}=\frac{1}{k_{n_{i}}}\left(1+I_{\Psi}\left(k_{n_{i}} f_{n_{i}}\right)\right) \\
& =\lim _{i \rightarrow \infty} \frac{1}{k_{n_{i}}}\left(1+I_{\Psi}\left(k_{n_{i}} f_{n_{i}}\right)\right) \\
& =\lim _{i \rightarrow \infty} \frac{1}{k_{n_{i}}} \int_{G} \Psi\left(k_{n_{i}} f_{n_{i}}(t)\right) d \mu \\
& \geqq \lim _{i \rightarrow \infty} \frac{1}{k_{n_{i}}} \int_{\left\{t \in G:\left|f_{n_{i}}(t)\right| \geqq a\right\}} \Psi\left(k_{n_{i}} f_{n_{i}}(t)\right) d \mu \\
& \geqq \lim _{i \rightarrow \infty} \frac{1}{k_{n_{i}}} \Psi\left(k_{n_{i}} a\right) \mu\left\{t \in G:\left|f_{n_{i}}(t)\right| \geqq a\right\} \\
& \geqq \lim _{i \rightarrow \infty} \frac{\Psi\left(k_{n_{i}}\right)}{k_{n_{i}}} \varepsilon=\infty,
\end{aligned}
$$


which is a contradiction. So, the sequence $\left\{k_{n}\right\}$ is bounded.

II. We shall prove that the functions of the sequence $\left\{f_{n}\right\}$ have equi-absolutely continuous norms. By $\Psi \in \Delta_{2}$, we only need to prove that

$$
\lim _{\mu(F) \rightarrow 0} \sup _{n} I_{\Psi}\left(f_{n} \chi_{F}\right)=0 .
$$

Otherwise, we can assume without loss of generality that

$$
I_{\Psi}\left(f_{n} \chi F_{n}\right) \geqq \varepsilon_{0} \quad \text { and } \quad \mu\left(F_{n}\right)<2^{-n} \mu(G)
$$

for a sequence $\left\{F_{n}\right\}$ of measurable subsets of $G$. Take a number $m \in \mathbb{N}$ such that

$$
\left\|f \chi_{G \backslash F}\right\|^{0} \geqq\|f\|^{0}-\varepsilon_{0} / 2=1-\varepsilon_{0} / 2
$$

whenever $\mu(F)<2^{-m} \mu(G)$. We have for $n>m$,

$$
\begin{aligned}
1 & =\left\|f_{n}\right\|^{0}=\frac{1}{k_{n}}\left(1+I_{\Psi}\left(k_{n} f_{n}\right)\right) \\
& =\frac{1}{k_{n}}\left(1+I_{\Psi}\left(k_{n} f_{n} \chi_{G \backslash \bigcup_{i>m} F_{i}}\right)+I_{\Psi}\left(k_{n} f_{n} \chi \bigcup_{i>m} F_{i}\right)\right) \\
& \geqq\left\|f_{n} \chi_{G \backslash \bigcup_{i>m} F_{i}}\right\|^{0}+I_{\Psi}\left(f_{n} \chi_{F_{n}}\right) \\
& \geqq\left\|f_{n} \chi_{G \backslash \bigcup_{i>m} F_{i}}\right\|^{0}+\varepsilon_{0} .
\end{aligned}
$$

Since $f_{n} \stackrel{E^{\Phi}}{\rightarrow} f$ by (i), we have

$$
\liminf _{n \rightarrow \infty}\left\|f_{n} \chi_{G \backslash \bigcup_{i>m} F_{i}}\right\|^{0} \geqq\left\|f \chi_{G \backslash \bigcup_{i>m} F_{i}}\right\|>1-\varepsilon_{0} / 2
$$

Hence, we get

$$
1 \geqq 1-\varepsilon_{0} / 2+\varepsilon_{0}=1+\varepsilon_{0} / 2,
$$

which is a contradiction. So, $f_{n}(n=1,2, \ldots)$ have equi-absolutely continuous norms.

III. We shall prove that $k_{n} f_{n}-k_{0} f \stackrel{\leftrightarrow}{\rightarrow} 0$. Otherwise, we can assume without loss of generality that there exist $\sigma_{0}>0$ and $\varepsilon_{0}>0$ such that

$$
\mu\left\{t \in G:\left|k_{n} f_{n}(t)-k_{0} f(t)\right| \geqq \sigma_{0}\right\} \geqq \varepsilon_{0}
$$

for any $n \in \mathbb{N}$. By step $\mathrm{I}$, we may assume that there is $d>1$ such that $1<k_{n} \leqq 1+d$ for $n=0,1,2 \ldots$ Define $k=\Psi^{-1}\left(3 / \varepsilon_{0}\right)(1+d)$ and

$$
G_{n}=\left\{t \in G:\left|k_{n} f_{n}(t)\right| \leqq k,\left|k_{0} f(t)\right| \leqq k \text { and }\left|k_{n} f(t)-k_{0} f(t)\right| \geqq \sigma_{0}\right\} .
$$


Since for any $u \in S\left(L_{0}^{\Psi}\right)$,

$$
\begin{aligned}
1 & \geqq \int_{G} \Psi(u(t)) d \mu \geqq \int_{\{t \in G:(1+d)|u(t)|>k\}} \Psi(u(t)) d \mu \\
& >\Psi\left(\frac{k}{1+d}\right) \mu(\{t \in G:(1+d)|u(t)|>k\})=\frac{3}{\varepsilon_{0}} \mu(\{t \in G:(1+d)|u(t)|>k\}),
\end{aligned}
$$

we get $\mu(\{t \in G:(1+d)|u(t)|>k\})<\varepsilon_{0} / 3$. Hence

$$
\begin{gathered}
\mu\left(G_{n}\right) \geqq \mu\left(\left\{t \in G:\left|k_{n} f_{n}(t)-k_{0} f(t)\right| \geqq \sigma_{0}\right\}\right)-\mu\left(\left\{t \in G:\left|k_{0} f(t)\right|>k\right\}\right) \\
-\mu\left(\left\{t \in G:\left|k_{n} f_{n}(t)\right|>k\right\}\right)>\varepsilon_{0}-\varepsilon_{0} / 3-\varepsilon_{0} / 3>\varepsilon_{0} / 3 .
\end{gathered}
$$

Since

$$
\begin{aligned}
& 0<\frac{1}{2+d}<\frac{k_{n}}{k_{n}+1+d} \leqq \frac{k_{n}}{k_{n}+k_{0}}<\frac{k_{n}}{k_{n}+1} \leqq \frac{1+d}{2+d}<1, \\
& 0<\frac{1}{2+d}<\frac{k_{0}}{k_{0}+1+d} \leqq \frac{k_{0}}{k_{n}+k_{0}} \leqq \frac{k_{0}}{k_{0}+1} \leqq \frac{1+d}{2+d}<1,
\end{aligned}
$$

by strict convexity of $\Psi$ (which is equivalent to smoothness of $\Phi$ ), there is $\delta>0$ such that for every $u, v$ with $|u| \leqslant k,|v| \leqslant k,|u-v| \geqq \sigma_{0}$ and for every $\alpha \in$ $[1 /(2+d),(1+d) /(2+d)]$, we have

$$
\Psi(\alpha u+(1-\alpha) v) \leqslant(1-\delta)\{\alpha \Psi(u)+(1-\alpha) \Psi(v)\} .
$$

Since $\left(f_{n}+f\right)(x) \rightarrow 2$, we have $\left\|f_{n}+f\right\|^{0} \rightarrow 2$. Noticing that $k_{n} /\left(k_{n}+k_{0}\right)$ $+k_{0} /\left(k_{n}+k_{0}\right)=1$, we have

$$
\begin{aligned}
2 & \left\|f_{n}+f\right\|^{0} \leqslant \\
\leqq & \frac{k_{n}+k_{0}}{k_{n} k_{0}}\left\{1+I_{\Psi}\left(\frac{k_{n} k_{0}}{k_{n}+k_{0}}\left(f_{n}+f\right)\right)\right\} \\
& \left.\quad+\int_{G \backslash G_{n}}\left[\frac{k_{0}}{k_{n}+k_{0}} \Psi\left(k_{n} f_{n}(t)\right)+\frac{k_{n}}{k_{n}+k_{0}} \Psi\left(k_{0} f(t)\right)\right] d \mu\right\} \\
= & \frac{1}{k_{n}}\left(1+I_{\Psi}\left(k_{n} f_{n}\right)\right)+\frac{1}{k_{0}}\left(1+I_{\Psi}\left(k_{0} f\right)\right) \\
& \quad-\delta \int_{G_{n}}\left[\frac{1}{k_{n}} \Psi\left(k_{n} f_{n}(t)\right)+\frac{1}{k_{0}} \Psi\left(k_{0} f(t)\right)\right] d \mu \\
\leqq & 2-\frac{\delta}{1+d} \int_{G_{n}}\left[\Psi\left(k_{n} f_{n}(t)\right)+\Psi\left(k_{0} f(t)\right)\right] d \mu \\
\leqq & 2-2 \delta \frac{1}{1+d} \int_{G_{n}} \Psi\left(\frac{1}{2} k_{n} f_{n}(t)-\frac{1}{2} k_{0} f(t)\right) d \mu \\
\leqq & 2-\frac{2 \delta}{1+d} \Psi\left(\frac{\sigma_{0}}{2}\right) \mu\left(G_{n}\right) \\
\leqq & 2-\frac{2 \delta}{1+d} \Psi\left(\frac{\sigma_{0}}{2}\right) \frac{\varepsilon_{0}}{3}
\end{aligned}
$$


a contradiction. So, we have proved that $k_{n} f-k_{0} f \stackrel{\mu}{\rightarrow} 0$.

Thus, by equi-absolute continuity of the norms of all functions from the sequence $\left\{f_{n}\right\}$ and by $k_{n} f_{n}-k_{0} f \stackrel{\mu}{\rightarrow} 0$, defining

$$
F_{n}=\left\{t \in G: \frac{6}{\varepsilon}\left|k_{n} f_{n}(t)-k_{0} f(t)\right| \geqslant \Phi^{-1}\left(\frac{1}{\mu(G)}\right)\right\}
$$

we have $\left\|\left(k_{n} f_{n}-k_{0} f\right) \chi_{G \backslash F_{n}}\right\|^{0} \leqq 2\left\|\left(k_{n} f_{n}-k_{0} f\right) \chi_{G \backslash F_{n}}\right\|<2 \cdot \varepsilon / 6=\varepsilon / 3$ for all $n \in \mathbb{N}$ and $\mu\left(F_{n}\right) \rightarrow 0$ as $n \rightarrow \infty$. Consequently, $\left\|k_{n} f_{n} \chi F_{n}\right\|^{0}<\varepsilon / 3$ and $\left\|k_{0} f \chi F_{n}\right\|^{0}<\varepsilon / 3$ for all $n \in \mathbb{N}$ large enough, say for $n \geqq n_{0}$. Hence $\left\|k_{n} f_{n}-k_{0} f\right\|^{0}<\varepsilon$ for $n \geqq n_{0}$, that is, $\left\|k_{n} f_{n}-k_{0} f\right\|^{0} \rightarrow 0$. So, $\left\|\left(k_{n} / k_{0}\right) f_{n}-f\right\|^{0} \rightarrow 0$ and consequently $\left\|\left(k_{n} / k_{0}\right) f_{n}\right\|^{0} \rightarrow$ $\|f\|^{0}$. Since $\left\|f_{n}\right\|^{0} \rightarrow\|f\|^{0}$, this yields $k_{n} \rightarrow k_{0}$ and finally $\left\|f_{n}-f\right\|^{0} \rightarrow 0$. So, the proof is complete.

THEOREM 2.2. An Orlicz space $L_{0}^{\Phi}$ is weakly strongly exposed if and only if $\Phi \in \Delta_{2}, \Psi \in \Delta_{2}$, and $\Phi$ is strictly convex.

Proof: Sufficiency. Suppose $\left\{x_{n}\right\}_{n=0}^{\infty}$ is a sequence in $S\left(L_{0}^{\Phi}\right)$ such that $f\left(x_{n}\right) \rightarrow$ $f\left(x_{0}\right)=1$ for some $f \in \operatorname{Grad}(x)$. Since, by $\Phi \in \Delta_{2}$ and $\Psi \in \Delta_{2}, L_{0}^{\Phi}$ is reflexive, and we conclude that there are a subsequence $\left\{x_{n_{i}}\right\}$ of $\left\{x_{n}\right\}$ and $x \in L_{0}^{\Phi}$ such that $x_{n_{i}} \stackrel{w}{\rightarrow} x$. In particular, $f\left(x_{n_{i}}\right) \rightarrow f(x)$. By $f\left(x_{n_{i}}\right) \rightarrow 1$, we have $f(x)=1$. Thus $f\left(x_{0}+x\right)=2$, whence $\left\|x_{0}+x\right\|=2$. Since $L_{0}^{\Phi}$ is strictly convex (see [1] and [13]), this yields $x=x_{0}$. So, $x_{n_{i}} \stackrel{w}{\rightarrow} x_{0}$ and by the double extract subsequence theorem, we get $x_{n} \stackrel{w}{\rightarrow} x_{0}$.

Necessity. By Theorems 1.1 and 2.1, we only need to prove that $\Psi \in \Delta_{2}$. Assume, for the contrary that $\Psi \notin \Delta_{2}$. Then there is a sequence $\left\{u_{n}\right\}_{n=1}^{\infty}$ of positive numbers such that $u_{n} \rightarrow+\infty$ as $n \rightarrow \infty$ and

$$
\Psi\left(\left(1+\frac{1}{n}\right) u_{n}\right) \geqslant 2^{n} \Psi\left(u_{n}\right) \quad(n=1,2, \ldots) .
$$

Since $u_{n} \rightarrow+\infty$ as $n \rightarrow \infty$, we can find (passing to a subsequence if necessary) a sequence $\left\{G_{n}\right\}_{n=1}^{\infty}$ of measurable pairwise disjoint sets in $G$ such that $\mu\left(G_{n}\right)=$ $1 /\left(2^{n} \Psi\left(u_{n}\right)\right)$. Define

$$
y=\sum_{n=1}^{\infty} u_{n} \chi G_{n} .
$$

Then $I_{\Psi}(y)=1$ and for any $\tau>0, I_{\Psi}((1+\tau) y)=+\infty$. Let $\left\{v_{n}\right\}_{n=1}^{\infty}$ be a sequence of positive numbers such that $u_{n} \in \partial \Phi\left(v_{n}\right)=\left[\Phi_{-}^{\prime}\left(v_{n}\right), \Phi_{+}^{\prime}\left(v_{n}\right)\right]$ and define

$$
x=\sum_{n=1}^{\infty} v_{n} \chi G_{n} .
$$


Then we have (see [4])

$$
\|x\|^{0}=\int_{G} x(t) y(t) d \mu .
$$

Define $x_{0}=x /\|x\|^{0}$. Then $x_{0} \in S\left(L_{0}^{\Phi}\right)$ and $y \in \operatorname{Grad}\left(x_{0}\right)$. Assume $A_{n}=\{t \in G$ : $|y(t)| \leqslant n\}$. Then $\lim _{n \rightarrow \infty}\left\|y \chi_{G \backslash A_{n}}\right\|^{0}=1$. By

$$
\left\|y \chi_{G \backslash A_{n}}\right\|^{0}=\sup \left\{\int_{G \backslash A_{n}} z(t) y(t) d \mu:\|z\|=1\right\}
$$

there exists a sequence $\left\{z_{n}\right\}$ in $S\left(L^{\Phi}\right)$ such that

$$
z_{n}=z_{n} \chi_{G \backslash A_{n}} \quad \text { and } \quad \int_{G \backslash A_{n}} z_{n}(t) y(t) d \mu \rightarrow 1=\left\langle x_{0}, y\right\rangle .
$$

Taking into account that, in view of the Vallée-Poussin theorem, all the functions from $\left\{z_{n}\right\}_{n=1}^{\infty}$ have equi-absolutely continuous integrals, we get by $\mu\left(G \backslash A_{n}\right) \rightarrow 0$ that

$$
\lim _{n \rightarrow \infty} \int_{G \backslash A_{n}} z_{n}(t) d \mu=0
$$

Hence

$$
\lim _{n \rightarrow \infty} \int_{G}\left(x(t)-z_{n}(t)\right) d \mu=\int_{G} x(t) d \mu>0,
$$

that is, $z_{n} \stackrel{w}{\rightarrow} x$, which means that $L_{0}^{\Phi}$ is not $W S E$, and the proof is complete.

\section{REFERENCES}

[1] S.T. Chen, Geometry of Orlicz Spaces, Dissertationes Math. (to appear).

[2] S.T. Chen, 'Smoothness of Orlicz spaces', Comment. Math. Prace Mat. 27 (1987), 49-58.

[3] S.T. Chen, Y.M. Lu and B.X. Wang, 'On the properties WM(CLUR) and (LKR) of Orlicz sequence spaces', Fasc. Math. 22 (1991).

[4] S.T. Chen, H. Hudzik and A. Kamińska, 'Support functionals and smooth points in Orlicz spaces equipped with the Orlicz norm', Math. Japon. 39 (1994), 271-279.

[5] J. Diestel, Geometry of Banach spaces - selected topics, Lecture Notes in Math. 485 (Springer-Verlag, Berlin, Heidelberg, New York, 1975).

[6] R. Grzģslewicz and H. Hudzik, 'Smooth points of Orlicz spaces equipped with Luxemburg norm', Math. Nachr. 155 (1992), 31-45.

[7] M.A. Krasnoselskiĭ and Ya.B. Rutickiǐ, Convex functions and Orlicz spaces, (translation) (Nordhoff Ltd., Groningen, 1961).

[8] W.A.J. Luxemburg, Banach function spaces, Thesis, (Delft, 1955).

[9] J. Musielak, Orlicz spaces and modular spaces, Lecture Notes in Math. 1034 (SpringerVerlag, Berlin, Heidelberg, New York, 1983). 
[10] B.B. Panda and O.P. Kapoor, 'Generalization of local uniform convexity of the norm', $J$. Math. Anal. Appl. 52 (1975), 300-308.

[11] B.B. Panda and O.P. Kapoor, 'Approximative compactness and continuity of metric projections', Bull. Austral. Math. Soc. 11 (1974), 47-55.

[12] R.R. Phelps, Convex functions, monotone operators and differentiability, Lecture Notes in Math. 1364 (Springer-Verlag, Berlin, Heidelberg, New York, 1989).

[13] M.M. Rao and Z.D. Ren, Theory of Orlicz spaces (Marcel Dekker Inc., New York, Basel, Hong Kong, 1991).

[14] T.F. Wang and S.T. Chen, 'Smoothness and differentiability of Orlicz spaces', Chinese J. Eng. Math. 14 (1987), 113-115.

Habin University of Sciences and Technology

Harbin

People's Republic of China

Habin University of Sciences and Technology

Harbin

People's Republic of China
Faculty of Mathematics and Computer Science Adam Mickiewicz University

Poznań

Poland 